\title{
BOUNDS FOR OWEN'S MULTILINEAR EXTENSION
}

\author{
JOSEP FREIXAS, ${ }^{*}$ Engineering School of Manresa and Technical University of Catalonia
}

\begin{abstract}
Owen's multilinear extension (MLE) of a game is a very important tool in game theory and particularly in the field of simple games. Among other applications it serves to efficiently compute several solution concepts. In this paper we provide bounds for the MLE. Apart from its self-contained theoretical interest, the bounds offer the means in voting system studies of approximating the probability that a proposal is approved in a particular simple game having a complex component arrangement. The practical interest of the bounds is that they can be useful for simple games having a tedious MLE to evaluate exactly, but whose minimal winning coalitions and minimal blocking coalitions can be determined by inspection. Such simple games are quite numerous.
\end{abstract}

Keywords: Simple games; Owen's multilinear extension; bounds; approximations

2000 Mathematics Subject Classification: Primary 91A12; 90B50; 90C59; 91 B12

\section{Introduction}

In this paper some bounds for the MLE introduced by Owen [16] are gathered. We consider the MLE function in the context of a binary decision rule (simple game) and an external prediction, which estimates the independent probability that each voter votes in favor of a certain proposal. Using the MLE, we can evaluate the probability of the proposal being approved.

A simple model for a voting system is a pair $(N, \mathcal{W})$, where $N=\{1,2, \ldots, n\}$ denotes the set of players or voters, subsets of $N$ are coalitions, and $\mathcal{W}$ denotes the set of winning coalitions. Subsets of $N$ that are not in $\mathcal{W}$ are called losing coalitions; the set of losing coalitions is denoted by $\mathcal{L}$. A simple game is defined to be monotonic: subsets of losing coalitions are again losing. A winning coalition $S$ is minimal if each proper subcoalition in $S$ is losing. We denote by $\mathbb{W}^{m}$ the set of minimal winning coalitions. A coalition $S$ is blocking if its complement $N \backslash S$ is losing. We denote by $\mathscr{B}$ the set of blocking coalitions, and we denote by $\mathscr{B}^{m}$ the set of minimal blocking coalitions. The complement of each minimal blocking coalition is a maximal losing coalition. A simple game is proper if $S \in \mathcal{W}$ implies that $N \backslash S \in \mathcal{L}$. A simple game is strong if $S \in \mathcal{L}$ implies that $N \backslash S \in \mathcal{W}$. A simple game is decisive if it is proper and strong. The unanimity game associated to coalition $S \neq \varnothing$ is the game $\left(N, \mathfrak{w}_{S}\right)$, where $S$ is the unique minimal winning coalition.

The dual game $\left(N, \mathcal{W}^{*}\right)$ of the game $(N, \mathcal{W})$ is defined by $S \in \mathcal{W}^{*}$ if and only if $S \in \mathcal{B}$. In particular, $(N, \mathcal{W})$ is proper if and only if $\left(N, \mathcal{W}^{*}\right)$ is strong, and $(N, \mathcal{W})$ is strong if and only

Received 4 January 2007; revision received 3 October 2007.

* Postal address: Department of Applied Mathematics 3, Engineering School of Manresa, Av. Bases de Manresa 61-73, E-08242 Manresa, Spain. Email address: josep.freixas@upc.edu 
if $\left(N, W^{*}\right)$ is proper. Thus, $\mathbb{W}=W^{*}$ if and only if $(N, \mathcal{W})$ is decisive. Furthermore, the dual game is idempotent, i.e. $\left(\mathcal{W}^{*}\right)^{*}=W^{W}$.

One natural way to construct a simple game is to assign a (nonnegative) real number weight to each voter, and declare a coalition winning precisely when its total weight meets or exceeds some predetermined quota. Formally, $(N, \mathcal{W})$ is weighted if there exists a vector of nonnegative numbers $w=\left(w_{1}, w_{2}, \ldots, w_{n}\right)$ and a quota $q$ such that

$$
\sum_{i \in S} w_{i} \geq q \quad \Longleftrightarrow \quad S \in \mathcal{W}
$$

For additional material on simple games, the reader is referred to [3], [6], [8], [18], [19], and [21] among others.

Given a simple game $(N, \mathcal{W})$, assume that a proposal $\operatorname{Pr}$ has to be submitted to the members of an assembly $N$. An outsider interested in the approval or the rejection of proposal $\mathrm{Pr}$ estimates the expectation of the proposal being approved according to his or her viewpoint. The outsider considers that each player ' $i$ ' has an independent a priori probability $p_{i}$ (or prediction of voter $i$ for proposal $\mathrm{Pr}$ ) of voting in favor of the proposal. The proposal is approved if and only if the set $S$ of members that vote for $P r$ is a winning coalition i.e. $S \in \mathcal{W}$, so that abstention or absence is allowed but it does not count for approving proposal $\mathrm{Pr}$. Then the probability of $\operatorname{Pr}$ being approved is

$$
f(\boldsymbol{p})=\sum_{S \in \mathcal{W}} \prod_{i \in S} p_{i} \prod_{i \notin S}\left(1-p_{i}\right),
$$

where $\boldsymbol{p}=\left(p_{1}, \ldots, p_{n}\right) \in[0,1]^{n}$. In other words, given a vector of probabilities $\boldsymbol{p}$ associated to $\operatorname{Pr}, f(\boldsymbol{p})$ gives the a priori probability of proposal $P r$ being approved or the game expectation of the proposal being approved. The MLE of a simple game $(N, \mathcal{W}),(1)$, was introduced by Owen [16] in the more general context of cooperative games. The function $f$, when the domain is restricted to $\boldsymbol{p} \in\{0,1\}^{n}$, is a pseudo-Boolean function; this class of functions is studied and related to game theory in [12]. The minimum possible value of $f(\boldsymbol{p})$ is 0 and the maximum possible value is 1 . For proper decisions, the minimum and maximum possible values of $f(\boldsymbol{p})$ can be attained. From an observer viewpoint with prediction $\boldsymbol{p}, f(\boldsymbol{p})$ measures the compliance of a decision rule, i.e. the ease with which the proposal can be approved. See [14] or [9] and [10] about this interpretation for $f(\boldsymbol{p})$. Moreover, if $f^{*}$ is the $\operatorname{MLE}$ of $\left(N, \boldsymbol{W}^{*}\right)$ then

$$
f^{*}(\boldsymbol{p})=1-f(1-\boldsymbol{p}) .
$$

The number of terms in (1) can be extremely large, up to $2^{n}-1$, which is the number of addends if each nonempty coalition is winning. This number does not reduce greatly if the game is proper, in fact, there are then up to $2^{n-1}$ addends. The complexity of calculations grows exponentially with the number of voters. Even with a small assembly the necessary computation time easily exceeds the bounds of a possible realization: in the case of $n$ voters we face an exponential complexity of order $2^{n}$. Despite the technical advances and enormous progress in computer power, this cannot solve the fundamental nature of the problem at hand, so that many voting games have not yet been evaluated exactly. One of the main motivations for using approximations for the MLE is that they significantly reduce the computation time and give conditions for which the bounds provided can give reasonably good approximations for $f$. 
Owen related the MLE with the Shapley-Shubik index $\phi[16]$ and the Penrose-BanzhafColeman index $\beta$ [17], where

$$
\begin{aligned}
\phi_{i} & =\int_{0}^{1} f_{i}(p, p, \ldots, p) \mathrm{d} p, \\
\beta_{i} & =f_{i}\left(\frac{1}{2}, \frac{1}{2}, \ldots, \frac{1}{2}\right),
\end{aligned}
$$

for all $i \in N$. The notation $f_{i}$ stands for the partial derivative of $f$ with respect to component $i$. Straffin [20] derived the following expression to compute $\beta_{i}$ by considering multiple integration on the unit cube $[0,1]^{n}$ and using Fubini's theorem for all $i$ :

$$
\beta_{i}=\int_{[0,1]^{n}} f_{i}\left(p_{1}, p_{2}, \ldots, p_{n}\right) \mathrm{d} p_{1} \mathrm{~d} p_{2} \cdots \mathrm{d} p_{n} .
$$

Coleman [5] suggested the 'power of a collectivity to act' - in his own terms - as a real number to be assigned to each simple game. Coleman's measure essentially leads to the structural decisiveness index studied in [2], $f\left(\frac{\mathbf{1}}{\mathbf{2}}\right)$, where $\frac{\mathbf{1}}{\mathbf{2}}=\left(\frac{1}{2}, \ldots, \frac{1}{2}\right)$. Carreras and Freixas (see [4, Theorem 10]) extended (4) for a large class of semivalues (see [7]), $\psi^{p}$ for $0 \leq p \leq 1$, those with binomial weighting coefficients $p_{k}=p^{k}(1-p)^{n-1-k}$ for $0 \leq k \leq n-1$, so that

$$
\psi_{i}^{p}=f_{i}(p, p, \ldots, p)
$$

Freixas and Puente (see [11, Proposition 3.2]) considered multibinary probabilistic values $\varphi$, a large class of probabilistic values (see [22]) defined by the weighting coefficients

$$
p_{S}^{i}=\prod_{j \in S} p_{j} \prod_{\substack{j \notin S \\ j \neq i}}\left(1-p_{j}\right) \quad \text { for } S \subseteq N \backslash\{i\},
$$

which have the form

$$
\varphi_{i}=f_{i}\left(p_{1}, p_{2}, \ldots, p_{n}\right) .
$$

Expression (7) has also been used in different contexts by Carreras [1], Freixas and Pons [9], [10], and Laruelle et al. [15].

In summary, formulae (3)-(7) show that there is a strong relationship between values for games and Owen's MLE. The approach on bounds proposed here can also be applied to obtain bounds for the values considered in formulae (3)-(7).

The rest of the paper is organized as follows. Prior to introducing the bounds, in Section 2 we discuss two forms of simplification for the proposed problem: the Boolean subgame, which sometimes allows us to reduce the number of components for the MLE; and complete simple games for which the sets of minimal winning coalitions and minimal blocking coalitions are easily derived. Section 3 is devoted to finding bounds based on the sets of minimal winning coalitions and minimal winning blocking coalitions. In Section 4 we deal with bounds based on the inclusion-exclusion principle. Conditional probability is used to find some alternative bounds in Section 5. In Section 6 we conclude the paper by the restriction to the homogeneous case in which all components are equal.

\section{The Boolean subgame}

Computation of the MLE for complex voting systems might be a formidable task (in fact, impracticable in some cases) unless an efficient method is used. Developing such methods is 
therefore of interest, and thus, it would be useful if we had a simple way of obtaining bounds. In this section we consider two significant cases that might considerably reduce the problem of finding bounds.

Given a simple game $(N, \mathcal{W})$ and the two disjoint subsets $Y$ and $Z$ of $N$, we can always consider $N^{\prime}=N \backslash(Y \cup Z)$ and the game $\left(N^{\prime}, W^{\prime}\right)$ defined, for $S \subseteq N^{\prime}$, by

$$
S \in \mathcal{W}^{\prime} \quad \Longleftrightarrow \quad S \cup Z \in \mathcal{W} \text {. }
$$

This game is the Boolean subgame of $(N, W)$ determined by $Y$ and $Z$; see [21, pp. 21-22] for further details.

The notion of a Boolean subgame includes the known concepts of a subgame ( $Y \subseteq N$ and $Z=\varnothing)$ and a reduced game $(Y=\varnothing$ and $Z \subseteq N)$. Intuitively a subgame results from assuming that everyone not in $N^{\prime}$ votes no, while the reduced game results from assuming that everyone not in $N^{\prime}$ votes yes. In general, a Boolean subgame corresponds to having a group of voters that always vote yes, together with a disjoint group of voters who will always vote no, and asking about the voting system induced on the remaining players.

It is clear that a general Boolean subgame can verify that $\varnothing \in \mathcal{W}^{\prime}$ (if $Z \in \mathcal{W}$ ) or $N^{\prime} \notin \mathcal{W}^{\prime}$ (if $N \backslash Y \notin W$ ). In both cases the vote of elements in $Y \cup Z$ determines whether the proposal is accepted or rejected, and so there is no reason to analyze the behavior of the remaining voters. This is why we will assume that $Y \subseteq N$ (the set of voters completely decided against the proposal) is such that $N \backslash Y \in \mathcal{W}$, and $Z \subseteq N$ (the set of voters completely decided in favor of the proposal) is such that $Z \notin W$.

Observe that if $f$ is the multilinear function of the game $(N, \mathcal{W})$ and $f^{\prime}$ is the multilinear function of the Boolean subgame determined by $Y$ and $Z$ (with the supposition mentioned above), then

$$
f^{\prime}(\overline{\boldsymbol{p}})=f\left(\mathbf{0}_{Z}, \mathbf{1}_{Y}, \boldsymbol{p}\right),
$$

where the vector $\left(\mathbf{0}_{Z}, \mathbf{1}_{Y}, \boldsymbol{p}\right)$ denotes the state vector in which the states of the components in $Z \subset N \backslash Y$ are all 0 , the states of the components in $Y \subset N \backslash Z$ are all 1, and the state of component $i$, with $i \notin Y \cup Z$, equals $p_{i}$; whereas $\overline{\boldsymbol{p}}$ denotes the restriction of $\boldsymbol{p}$ to the components in $N^{\prime}$.

In voting systems, prior to the submission of a particular proposal, it is common for some voters to show their willingness to vote in favor of the proposal and others to show their willingness to vote against the proposal. In these situations we may considerably reduce the calculation of $f$ by considering the Boolean subgame.

As we shall see in the following sections, the proposed bounds are derived from the sets of minimal winning coalitions and minimal blocking coalitions. Thus, it is possible to calculate bounds computationally whenever it is possible to list these two sets for any game. Although some games have large sets $\mathfrak{W}^{m}$ and $\mathscr{B}^{m}$, there are subclasses of simple games for which it is easy to generate these sets. Indeed, a classification theorem by Carreras and Freixas [3, Theorem 4.1], allows us to generate these lists and count all complete (or linear) simple games up to isomorphism. Complete simple games are those for which the desirability relation,

$$
i \succsim_{D} j \quad \text { if and only if } S \cup\{j\} \in \mathcal{W} \Longrightarrow S \cup\{i\} \in \mathcal{W} \quad \text { for all } S \subseteq N \backslash\{i, j\},
$$

introduced by Isbell [13], is complete (or total). Using linear programming, we can obtain within the set of complete simple games the set of those which are weighted simple games. See the data of Table 1. 
TABLE 1: Number of complete games (CG), number of weighted games (WG), and the CPU time (in seconds) needed to compute all complete simple games with $n$ voters.

\begin{tabular}{ccccccccc}
\hline$n$ & 1 & 2 & 3 & 4 & 5 & 6 & 7 & 8 \\
\hline CG & 1 & 3 & 8 & 25 & 117 & 1171 & 44313 & 16175188 \\
WG & 1 & 3 & 8 & 25 & 117 & 1111 & 29373 & 2730164 \\
CPU time & $<1$ & $<1$ & $<1$ & $<1$ & $<1$ & $<1$ & 3 & $66532(\approx 18.5$ hours $)$ \\
\hline
\end{tabular}

The numbers of complete and weighted games exhibit an exponential growth. However, the classification theorem in [3] allows for each complete simple game to generate the sets $\mathbb{W}^{m}$ and $\mathscr{B}^{m}$ by providing only a vector with less than or equal to $n$ components and a matrix fulfilling some simple conditions. In other words, for any complete simple game and whenever $n$ is not too large, it is always possible to introduce a brief list of numbers into the computational program in order to generate the sets $W^{m}$ and $\mathscr{B}^{m}$ and, subsequently, to compute the bounds provided in Sections 3, 4, 5, and 6 .

\section{Bounds based on the sets of minimal winning coalitions and minimal blocking coalitions}

In Sections 3, 4, and 5 we outline some methods to approximate $f$, which are based on standard probability techniques. Some initial recommendable bounds for a voting system with a high number of voters are given by the following inequalities.

Proposition 1. Let $f$ be the MLE of a game $(N, W)$, and $\boldsymbol{p}$ be a predictions'vector. Then

$$
\begin{gathered}
\max _{S \in \mathcal{W}^{m}} \prod_{i \in S} p_{i} \leq f \leq 1-\max _{T \in \mathcal{B}^{m}} \prod_{i \in T} q_{i}, \\
\prod_{i \in S^{\prime}} p_{i}+\sum_{\substack{S \in \mathcal{W}^{m} \\
S \neq S^{\prime}}} \prod_{i \in S} p_{i} \prod_{i \notin S} q_{i} \leq f \leq 1-\left(\prod_{i \in T^{\prime}} q_{i}+\sum_{\substack{T \in \mathcal{B}^{m} \\
T \neq T^{\prime}}} \prod_{i \in T} q_{i} \prod_{i \notin T} p_{i}\right),
\end{gathered}
$$

where $q_{i}=1-p_{i}$ for each $1 \leq i \leq n$, and $S^{\prime}$ and $T^{\prime}$ are the coalitions which respectively attain

$$
\max _{S \in \mathcal{W}^{m}} \prod_{i \in S} p_{i} \text { and } \max _{T \in \mathcal{B}^{m}} \prod_{i \in T} q_{i} .
$$

Proof. The left bound in (8) is obtained by observing that $\mathcal{W}=\mathfrak{W}_{S_{1}} \cup \cdots \cup \mathcal{W}_{S_{m}}$, where $S_{1}, \ldots, S_{m}$ are the minimal winning coalitions of game $(N, \mathcal{W})$, and that $f$ associated to the unanimity game $\mathfrak{W}_{S_{j}}$ has $2^{\left|N \backslash S_{j}\right|}$ addends in (1) with total sum $\prod_{i \in S_{j}} p_{i}$. Thus, the term on the left-hand side of (8) is derived by taking the maximum of these products on $S_{1}, \ldots, S_{m}$. The right bound in (8) is derived using the left bound and the dual expression for the MLE, (2).

The two bounds in (8) are improved in (9) by incorporating the addends in (1) corresponding to the remaining minimal winning coalitions and minimal blocking coalitions, respectively.

Approximation (8) usually leads to very wide intervals for the probability to approve proposal $\operatorname{Pr}$. However, some particular cases suggest making use of it. For example, if the game has only a single winning coalition then the left bound becomes an equality. Alternatively, if the game has only a single minimal blocking coalition then the right bound becomes an equality. Thus, in these two cases the exact value for $f$ is attained. In general, if $\left|\mathfrak{W}^{m}\right| /|\mathfrak{W}|$ and $\left|\mathscr{B}^{m}\right| /|\mathscr{B}|$ are 
small enough then the left bound and the right bound, respectively, provide quite good initial approximations for $f$. Let us consider an elementary example, which becomes even simpler upon using the Boolean subgame.

Example 1. Consider the simple game with five voters and weighted representation $[9 ; 5,4,3$, $2,1]$. This game has four minimal winning coalitions, four minimal blocking coalitions, 13 winning coalitions, and 19 losing coalitions. If $\boldsymbol{p}=\left(\frac{4}{4}, \frac{3}{4}, \frac{2}{4}, \frac{1}{4}, \frac{0}{4}\right)$ is a predictions' vector for an outsider, the bounds in (8) for the associated Boolean subgame yield

$$
\frac{24}{32} \leq f \leq \frac{26}{32}
$$

The bounds in (9) yield

$$
\frac{25}{32} \leq f \leq \frac{26}{32}
$$

Note that the exact value for $f$ is $\frac{25}{32}$.

\section{Inclusion-exclusion bounds}

Often it is more efficient to let the starting point of the calculation be the complement of the predictions. Let $B_{j}$ be the event that all players in the minimal blocking coalition $T_{j}$ vote against proposal $\operatorname{Pr}, j=1,2, \ldots, k$, where $k$ is the number of minimal blocking coalitions. Then clearly

$$
\mathrm{P}\left(B_{j}\right)=\prod_{i \in T_{j}} q_{i}
$$

and

$$
1-f(\boldsymbol{p})=\mathrm{P}\left(\bigcup_{j=1}^{k} B_{j}\right)
$$

Furthermore, let

$$
\begin{aligned}
b_{1} & =\sum_{j=1}^{k} \mathrm{P}\left(B_{j}\right), \\
b_{2} & =\sum_{1 \leq i<j \leq k}^{k} \mathrm{P}\left(B_{i} \cap B_{j}\right), \\
& \vdots \\
b_{r} & =\sum_{1 \leq i_{1}<i_{2}<\cdots<i_{r} \leq k} \mathrm{P}\left(\bigcap_{j=1}^{r} B_{i_{j}}\right) .
\end{aligned}
$$

Then the well-known inclusion-exclusion formula states that

$$
1-f(\boldsymbol{p})=b_{1}-b_{2}+\cdots+(-1)^{k+1} b_{k}
$$

Equality (10) can be proven by induction on the number of events $B_{j}$. The following result is interesting for our purposes. 
Proposition 2. Let $(N, W)$ be a simple game with $f$ as an MLE, let $\boldsymbol{p}$ be a predictions'vector, and let $b_{1}, \ldots, b_{r}$ be defined as above with $r \leq k$. Then

$$
\begin{aligned}
& 1-f(\boldsymbol{p}) \leq b_{1}-b_{2}+\cdots+b_{r} \quad \text { if } r \text { is odd } \\
& 1-f(\boldsymbol{p}) \geq b_{1}-b_{2}+\cdots-b_{r} \quad \text { if } r \text { is even. }
\end{aligned}
$$

Proof. Consider the indicator variables $I_{j}, j=1, \ldots, k$, defined as

$$
I_{j}= \begin{cases}1 & \text { if } B_{j} \text { occurs } \\ 0 & \text { otherwise }\end{cases}
$$

Letting $M=\sum_{j=1}^{k} I_{j}$, then $M$ denotes the number of $B_{j}, 1 \leq j \leq k$, that occur. Also, let

$$
I= \begin{cases}1 & \text { if } M>0 \\ 0 & \text { if } M=0\end{cases}
$$

Then, as $1-I=(1-1)^{M}$ (here $\left.0^{0}=1\right)$, we obtain, upon application of the binomial theorem,

$$
1-I=\sum_{i=0}^{M}\left(\begin{array}{c}
M \\
i
\end{array}\right)(-1)^{i}=1-M+\left(\begin{array}{c}
M \\
2
\end{array}\right)-\left(\begin{array}{c}
M \\
3
\end{array}\right)+\cdots \pm\left(\begin{array}{c}
M \\
M
\end{array}\right) .
$$

We now make use of the following combinatorial identity (which is easily established by induction on $i$ ):

$$
\left(\begin{array}{l}
k \\
i
\end{array}\right)-\left(\begin{array}{c}
k \\
i+1
\end{array}\right)+\cdots \pm\left(\begin{array}{l}
k \\
k
\end{array}\right)=\left(\begin{array}{l}
k-1 \\
i-1
\end{array}\right) \geq 0, \quad i \leq k .
$$

The preceding thus implies that

$$
\left(\begin{array}{c}
M \\
i
\end{array}\right)-\left(\begin{array}{c}
M \\
i+1
\end{array}\right)+\cdots \pm\left(\begin{array}{c}
M \\
M
\end{array}\right) \geq 0
$$

From (12) and (13), we obtain

$$
\begin{array}{ll}
I \leq M, & \text { by letting } i=2 \text { in (13), } \\
I \geq M-\left(\begin{array}{c}
M \\
2
\end{array}\right), & \text { by letting } i=3 \text { in (13), } \\
I \leq M-\left(\begin{array}{c}
M \\
2
\end{array}\right)+\left(\begin{array}{c}
M \\
3
\end{array}\right), &
\end{array}
$$

and so on. Now, since $M \leq k$ and $\left(\begin{array}{l}k \\ i\end{array}\right)=0$ whenever $i>k$, we can simplify (12) as

$$
I=\sum_{i=1}^{k}\left(\begin{array}{c}
M \\
i
\end{array}\right)(-1)^{i+1}
$$


Equality (10) and inequalities (11) now follow upon taking expectations of (14) and (15). This is the case since

$$
\begin{gathered}
\mathrm{E}[I]=\mathrm{P}\{M>0\}=\mathrm{P}\left\{\text { at least one of the } B_{j} \text { occurs }\right\}=\mathrm{P}\left(\bigcup_{j=1}^{k} B_{j}\right), \\
\mathrm{E}[M]=\mathrm{E}\left[\sum_{j=1}^{k} I_{j}\right]=\sum_{j=1}^{k} \mathrm{P}\left(B_{j}\right) .
\end{gathered}
$$

Also,

$$
\begin{aligned}
\mathrm{E}\left[\left(\begin{array}{c}
M \\
i
\end{array}\right)\right] & =\mathrm{E}[\text { number of sets of size } i \text { that occur }] \\
& =\mathrm{E}\left[\sum_{1 \leq i_{1}<i_{2}<\cdots<i_{r} \leq k} I_{i_{1}} I_{i_{2}} \cdots I_{i_{r}}\right] \\
& =\sum_{1 \leq i_{1}<i_{2}<\cdots<i_{r} \leq k} \mathrm{P}\left(\bigcap_{j=1}^{r} B_{i_{j}}\right) .
\end{aligned}
$$

Although in general it is not true that the upper bounds decrease and the lower bounds increase, in practice it may be necessary to calculate only a few $b_{r}$ terms to obtain a close approximation. If each $q_{i}$ is small, i.e. the predictions for each voter are large, then the $b_{2}$ term will be negligible compared to $b_{1}$; thus, $1-f \approx b_{1}$. Note that $b_{1}$ is an upper bound for $1-f$, so the approximation $f \approx 1-b_{1}$ produces an underestimation of $f$. The number of terms in the sum $b_{r}$ equals $\left(\begin{array}{l}k \\ r\end{array}\right)$. Thus, the total number of terms in the expression of $1-f$ equals $2^{k}-1$ ( $k$ is the number of minimal blocking coalitions).

Alternatively, if each $p_{i}$ is small then we may repeat the same argument considering $W_{j}$, the event that all the players in the minimal winning coalition $S_{j}$ vote for proposal $\operatorname{Pr}, j=$ $1,2, \ldots, m$, where $m$ is the number of minimal winning coalitions. Then,

$$
\mathrm{P}\left(W_{j}\right)=\prod_{i \in S_{j}} p_{i}
$$

and

$$
f(\boldsymbol{p})=\mathrm{P}\left(\bigcup_{j=1}^{m} W_{j}\right) .
$$

Furthermore, let

$$
\begin{aligned}
w_{1} & =\sum_{j=1}^{k} \mathrm{P}\left(W_{j}\right), \\
w_{2} & =\sum_{1 \leq i<j \leq m}^{k} \mathrm{P}\left(W_{i} \cap W_{j}\right), \\
& \vdots \\
w_{r}= & \sum_{1 \leq i_{1}<i_{2}<\cdots<i_{r} \leq m} \mathrm{P}\left(\bigcap_{j=1}^{r} W_{i_{j}}\right) .
\end{aligned}
$$


Then the inclusion-exclusion formula states that

$$
f(\boldsymbol{p})=w_{1}-w_{2}+\cdots+(-1)^{k+1} w_{m},
$$

and, for $r \leq m$,

$$
\begin{array}{ll}
f(\boldsymbol{p}) \leq w_{1}-w_{2}+\cdots+w_{r} & \text { if } r \text { is odd } \\
f(\boldsymbol{p}) \geq w_{1}-w_{2}+\cdots-w_{r} & \text { if } r \text { is even. }
\end{array}
$$

If each $p_{i}$ is small, i.e. the predictions for each voter are small, then the $w_{2}$ term will be negligible compared to $w_{1}$; thus, $f \approx w_{1}$. Note that $w_{1}$ is an upper bound for $f$, so the approximation $f \approx w_{1}$ produces an overestimation of $f$.

\section{Bounds based on conditional probability}

An alternative way to approximate $f$ is now presented.

Proposition 3. Let $S_{1}, \ldots, S_{m}$ denote the minimal winning coalitions of the game $(N, \mathcal{W})$, and let $T_{1}, \ldots, T_{k}$ denote the minimal blocking coalitions, then

$$
\prod_{j=1}^{k}\left(1-\prod_{i \in T_{j}} q_{i}\right) \leq f \leq 1-\prod_{j=1}^{m}\left(1-\prod_{i \in S_{j}} p_{i}\right)
$$

Proof. Let $S_{1}, S_{2}, \ldots, S_{m}$ denote the minimal winning coalitions of the game $(N, \mathcal{W})$, and define the events $A_{1}, \ldots, A_{m}$ by $A_{j}$ 'at least one voter in $S_{j}$ votes against proposal $P r$ '. Now

$$
\begin{aligned}
1-f(\boldsymbol{p}) & =\mathrm{P}\left(A_{1} \cap A_{2} \cap \cdots \cap A_{m}\right) \\
& =\mathrm{P}\left(A_{1}\right) \mathrm{P}\left(A_{2} \mid A_{1}\right) \cdots \mathrm{P}\left(A_{m} \mid A_{1} \cap A_{2} \cap \cdots \cap A_{m-1}\right) .
\end{aligned}
$$

Now we are going to show that $\mathrm{P}\left(A_{2} \mid A_{1}\right) \geq \mathrm{P}\left(A_{2}\right)$. To prove this inequality, we make use of the conditional probability

$$
\mathrm{P}\left(A_{2}\right)=\mathrm{P}\left(A_{2} \mid A_{1}\right) \mathrm{P}\left(A_{1}\right)+\mathrm{P}\left(A_{2} \mid A_{1}^{c}\right) \mathrm{P}\left(A_{1}^{c}\right),
$$

and note that

$$
\begin{aligned}
\mathrm{P}\left(A_{2} \mid A_{1}^{c}\right) & =1-\prod_{i \in S_{2} \cap S_{1}^{c}} p_{i} \\
& \leq 1-\prod_{i \in S_{2}} p_{i} \\
& =\mathrm{P}\left(A_{2}\right) .
\end{aligned}
$$

Hence,

$$
\mathrm{P}\left(A_{2}\right) \leq \mathrm{P}\left(A_{2} \mid A_{1}\right) \mathrm{P}\left(A_{1}\right)+\mathrm{P}\left(A_{2}\right)\left(1-\mathrm{P}\left(A_{1}\right)\right),
$$

or, equivalently,

$$
\mathrm{P}\left(A_{2} \mid A_{1}\right) \geq \mathrm{P}\left(A_{2}\right) .
$$


TABLE 2: Lower and upper bounds for some symmetric games.

\begin{tabular}{|c|c|c|c|c|}
\hline \multicolumn{2}{|c|}{ Game } & \multicolumn{3}{|c|}{ Bounds } \\
\hline$n$ & $d$ & (8) & (9) & (16) \\
\hline \multicolumn{5}{|c|}{ Lower bounds } \\
\hline 4 & 3 & 0.9310950 & 0.9955193 & 0.9954069 \\
\hline 8 & 6 & 0.8072015 & 0.8112102 & 0.9996939 \\
\hline 16 & 12 & 0.7515813 & 0.7524603 & 0.9999237 \\
\hline \multicolumn{5}{|c|}{ Upper bounds } \\
\hline 4 & 3 & 0.9975000 & 0.9955288 & 0.9999461 \\
\hline 8 & 6 & 0.9999938 & 0.9997485 & $\approx 1$ \\
\hline 16 & 12 & 0.9999997 & 0.9999423 & $\approx 1$ \\
\hline
\end{tabular}

Using the same argument, it also follows that

$$
\mathrm{P}\left(A_{j} \mid A_{1} \cap A_{2} \cap \cdots \cap A_{j-1}\right) \geq \mathrm{P}\left(A_{j}\right),
$$

and so we have

$$
1-f(\boldsymbol{p}) \geq \prod_{j=1}^{m} \mathrm{P}\left(A_{j}\right),
$$

or, equivalently,

$$
f(\boldsymbol{p}) \leq 1-\prod_{j=1}^{m}\left(1-\prod_{i \in S_{j}} p_{i}\right) .
$$

The bound in the other direction follows upon applying duality, (2), to the right-hand side bound in (17). Thus, (16) is proved.

It is to be expected that the upper bound should be close to the actual value of $f(\boldsymbol{p})$ if there is not too much overlap in the minimal winning coalitions (for example, minimal winning coalitions in improper games do not overlap much), and the lower bound should be close to the exact value of $f(\boldsymbol{p})$ if there is not too much overlap in the minimal blocking coalitions. The bounds obtained are also good approximations for either small values or large values of the $p_{i} \mathrm{~s}$.

Example 2. Consider symmetric games (that is, weighted games with the same weight assigned to each voter) with $n$ players and a demand of $75 \%$ of the membership. The winning coalitions are those having at least $75 \%$ of the members in $N$. We consider the cases in which $n=4,8$, and 16 with respective demands $d=3,6$, and 12. For instance, if $p_{1}=\cdots=p_{n / 2}=0.99$ and $p_{n / 2+1}=\cdots=p_{n}=0.95$ for $n=4,8$, and 16, the bounds provided by (8), (9), and (16) are given in Table 2 . The figures in the boxes represent the best lower and upper bounds found.

However, since each $q_{i}$ is small, the best bounds are obtained using (11). For instance, if we take $r=1$ and $r=2$, we obtain $0.9999385 \leq f \leq 0.99994$ for $n=4$ and $d=3$, which is a better approximation for $f$ than those given in Table 2 . 


\section{Bounds for the homogeneous case}

If $p_{i}=p$ for every voter $i$, the MLE given in (1) may be simplified to obtain a function of the type

$$
f(\boldsymbol{p})=\sum_{i=0}^{n} A_{i} p^{i}(1-p)^{n-i},
$$

where $n$ denotes the number of voters, and $A_{i}$ denotes the number of winning coalitions with $i$ members which satisfies $A_{i} \leq\left(\begin{array}{c}n \\ i\end{array}\right)$ with $i=0,1, \ldots, n$. This last inequality simply reflects the fact that there are at most $\left(\begin{array}{l}n \\ i\end{array}\right)$ coalitions of size $i$. For the homogeneous case and when $p$ ranges between 0 and 1 , the lower and upper bounds define functions $f_{\mathrm{L}}(p)$ and $f_{\mathrm{U}}(p)$, respectively.

Many real-world voting systems are $d$-out-of- $n$ games, i.e. voting systems in which the proposal at hand passes if at least $d$ of its $n$ members vote in favor of it. A $d$-out-of- $n$ game is proper if and only if $d>n / 2$ and strong if and only if $d<1+n / 2$, and, therefore, decisive if and only if $n$ is odd and $d=(n+1) / 2$. For $d$-out-of- $n$ games, the calculation of $f$ still becomes a complex task if $n$ is large enough. Let us provide some bounds for $d$-out-of- $n$ games using the results derived in Section 5. The bounds provided in (8) yield $f_{\mathrm{L}}(p)=p^{d}$ and $f_{\mathrm{U}}(p)=1-(1-p)^{n-d+1}$. The bounds given in (9) yield

$$
\begin{aligned}
& f_{\mathrm{L}}(p)=p^{d}+\left(\left(\begin{array}{l}
n \\
d
\end{array}\right)-1\right) p^{d}(1-p)^{n-d} \text { and } \\
& f_{\mathrm{U}}(p)=1-\left((1-p)^{n-d+1}+\left(\begin{array}{c}
n \\
n-d+1
\end{array}\right)(1-p)^{n-d+1} p^{d-1}\right) .
\end{aligned}
$$

The bounds given in (16) yield the following expressions, which are easy to evaluate:

$$
f_{\mathrm{L}}(p)=\left(1-(1-p)^{n-d+1}\right)^{\left(\begin{array}{c}
n \\
n-d+1
\end{array}\right)} \text { and } \quad f_{\mathrm{U}}(p)=1-\left(1-p^{d}\right)^{\left(\begin{array}{c}
n \\
d
\end{array}\right)}
$$

See Figure 1 for $d=3$ and $n=4$, where we observe that $f_{L}(p) \leq f(p) \leq f_{U}(p)$ for all $p \in[0,1]$.

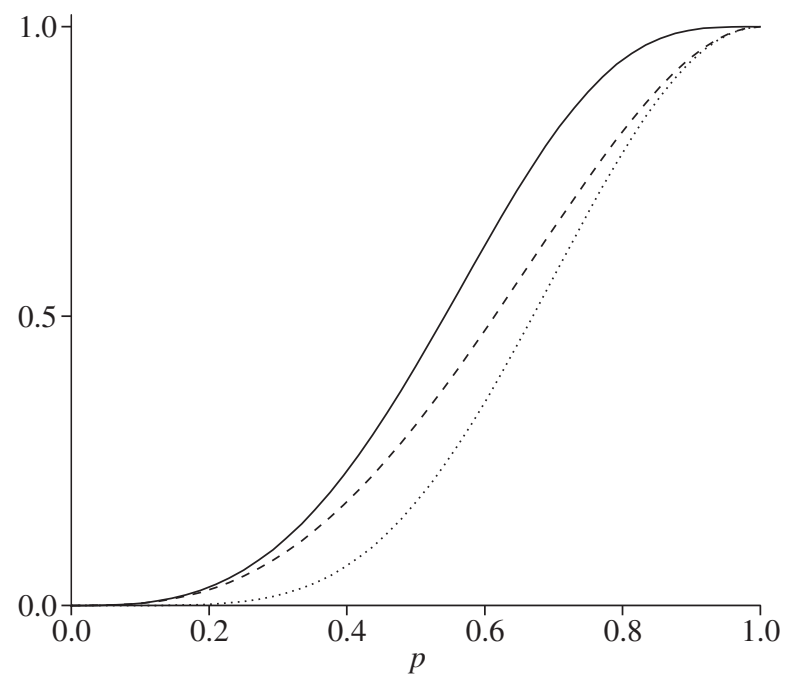

FIGURE 1: Bounds (18) for the 3-out-of-4 simple game. 
TABLE 3: The bounds $f_{\mathrm{L}}(p)$ and $f_{\mathrm{U}}(p)$ given by (16) for all simple games with $n \leq 4$ players.

\begin{tabular}{ccccc}
\hline Game & $\boldsymbol{W}^{m}$ & $\mathcal{B}^{m}$ & $f_{\mathrm{L}}(p)$ & $f_{\mathrm{U}}(p)$ \\
\hline 1 & $\{1\}$ & $\{1\}$ & $(1-q)$ & $1-(1-p)$ \\
2 & $\{1 ; 2\}$ & $\{12\}$ & $\left(1-q^{2}\right)$ & $1-(1-p)^{2}$ \\
3 & $\{1 ; 2 ; 3\}$ & $\{123\}$ & $\left(1-q^{3}\right)$ & $1-(1-p)^{3}$ \\
4 & $\{1 ; 2 ; 3 ; 4\}$ & $\{1234\}$ & $\left(1-q^{4}\right)$ & $1-(1-p)^{4}$ \\
5 & $\{1 ; 2 ; 34\}$ & $\{123 ; 124\}$ & $\left(1-q^{3}\right)^{2}$ & $1-(1-p)^{2}\left(1-p^{2}\right)$ \\
6 & $\{1 ; 23\}$ & $\{12 ; 13\}$ & $\left(1-q^{2}\right)^{2}$ & $1-(1-p)\left(1-p^{2}\right)$ \\
7 & $\{1 ; 23 ; 24\}$ & $\{12 ; 134\}$ & $\left(1-q^{2}\right)\left(1-q^{3}\right)$ & $1-(1-p)\left(1-p^{2}\right)^{2}$ \\
8 & $\{1 ; 23 ; 24 ; 34\}$ & $\{123 ; 124 ; 134\}$ & $\left(1-q^{3}\right)^{3}$ & $1-(1-p)\left(1-p^{2}\right)^{3}$ \\
9 & $\{1 ; 234\}$ & $\{12 ; 34\}$ & $\left(1-q^{2}\right)^{2}$ & $1-(1-p)\left(1-p^{3}\right)$ \\
10 & $\{12\}$ & $\{1 ; 2\}$ & $(1-q)^{2}$ & $1-\left(1-p^{2}\right)$ \\
11 & $\{12 ; 13\}$ & $\{1 ; 23\}$ & $(1-q)\left(1-q^{2}\right)$ & $1-\left(1-p^{2}\right)^{2}$ \\
12 & $\{12 ; 13 ; 14\}$ & $\{12 ; 13 ; 24 ; 34\}$ & $\left(1-q^{2}\right)^{4}$ & $1-\left(1-p^{2}\right)^{3}$ \\
13 & $\{12 ; 13 ; 14 ; 23\}$ & $\{12 ; 13 ; 234\}$ & $\left(1-q^{2}\right)^{2}\left(1-q^{3}\right)$ & $1-\left(1-p^{2}\right)^{4}$ \\
14 & $\{12 ; 13 ; 14 ; 23 ; 24\}$ & $\{12 ; 134 ; 234\}$ & $\left(1-q^{2}\right)\left(1-q^{3}\right)^{2}$ & $1-\left(1-p^{2}\right)^{5}$ \\
15 & $\{12 ; 13 ; 14 ; 23 ; 24 ; 34\}$ & $\{123 ; 124 ; 134 ; 234\}$ & $\left(1-q^{3}\right)^{4}$ & $1-\left(1-p^{2}\right)^{6}$ \\
16 & $\{12 ; 13 ; 14 ; 234\}$ & $\{12 ; 13 ; 14 ; 234\}$ & $\left(1-q^{2}\right)^{3}\left(1-q^{3}\right)$ & $1-\left(1-p^{2}\right)^{3}\left(1-p^{3}\right)$ \\
17 & $\{12 ; 13 ; 23\}$ & $\{12 ; 13 ; 23\}$ & $\left(1-q^{2}\right)^{3}$ & $1-\left(1-p^{2}\right)^{3}$ \\
18 & $\{12 ; 13 ; 24\}$ & $\{12 ; 13 ; 24\}$ & $\left(1-q^{2}\right)^{3}$ & $1-\left(1-p^{2}\right)^{3}$ \\
19 & $\{12 ; 13 ; 24 ; 34\}$ & $\{12 ; 13 ; 14\}$ & $\left(1-q^{2}\right)^{3}$ & $1-\left(1-p^{2}\right)^{4}$ \\
20 & $\{12 ; 13 ; 234\}$ & $\{12 ; 13 ; 14 ; 23\}$ & $\left(1-q^{2}\right)^{4}$ & $1-\left(1-p^{2}\right)^{2}\left(1-p^{3}\right)$ \\
21 & $\{12 ; 34\}$ & $\{1 ; 234\}$ & $(1-q)\left(1-q^{3}\right)$ & $1-\left(1-p^{2}\right)^{2}$ \\
22 & $\{12 ; 134\}$ & $\{1 ; 23 ; 24\}$ & $(1-q)\left(1-q^{2}\right)^{2}$ & $1-\left(1-p^{2}\right)\left(1-p^{3}\right)$ \\
23 & $\{12 ; 134 ; 234\}$ & $\{12 ; 13 ; 14 ; 23 ; 24\}$ & $\left(1-q^{2}\right)^{5}$ & $1-\left(1-p^{2}\right)\left(1-p^{3}\right)^{2}$ \\
24 & $\{123\}$ & $\{1 ; 2 ; 3\}$ & $(1-q)^{3}$ & $1-\left(1-p^{3}\right)$ \\
25 & $\{123 ; 124\}$ & $\{1 ; 2 ; 34\}$ & $(1-q)^{2}\left(1-q^{2}\right)$ & $1-\left(1-p^{3}\right)^{2}$ \\
26 & $\{123 ; 124 ; 134\}$ & $\{1 ; 23 ; 24 ; 34\}$ & $(1-q)\left(1-q^{2}\right)^{3}$ & $1-\left(1-p^{3}\right)^{3}$ \\
27 & $\{123 ; 124 ; 134 ; 234\}$ & $\{12 ; 13 ; 14 ; 23 ; 24 ; 34\}$ & $\left(1-q^{2}\right)^{6}$ & $1-\left(1-p^{3}\right)^{4}$ \\
28 & $\{1234\}$ & $\{1 ; 2 ; 3 ; 4\}$ & $(1-q)^{4}$ & $1-\left(1-p^{4}\right)$ \\
\hline & & & &
\end{tabular}

Table 3 contains data on all simple games with $n \leq 4$ players, where the lower bound, $f_{\mathrm{L}}(p)$, and the upper bound, $f_{\mathrm{U}}(p)$, are given by (16) for the homogeneous case. It is important to note that these functions defined on the respective sets $W^{m}$ and $\mathscr{B}^{m}$ depend only on the numbers of coalitions for each size in their respective sets. For instance, games 17 and 18 both have three coalitions of size 2 in sets $\mathfrak{W}^{m}$ and $\mathscr{B}^{m}$, so their respective bounds coincide.

Also note that the four symmetric games (or $d$-out-of- $n$ games) are games 4, 15, 27, and 28, which respectively follow from formulae (18). The lower and the upper bounds coincide for games with a single element either in $\mathcal{W}^{m}$ or in $\mathscr{B}^{m}$; this is the case for games $1,2,3,4,10$, 24 , and 28. Hence, for these games we have the exact value of $f$.

\section{Acknowledgements}

The author wishes to thank two anonymous referees for carefully reading an earlier version of this paper and pointing out useful comments that improved its presentation. This research was partially supported by Grant MTM 2006-06064 of 'Ministerio de Ciencia y Tecnología' and 'Fondo Europeo de Desarrollo Regional' and SGRC 2005-00651 of 'Generalitat de Catalunya'. 


\section{References}

[1] Carreras, F. (2004). $\alpha$-decisiveness in simple games. Theory Decision 56, 77-91.

[2] Carreras, F. (2005). A decisiveness index for simple games. Europ. J. Operat. Res. 163, 370-387.

[3] Carreras, F. and Freixas, J. (1996). Complete simple games. Math. Social Sci. 32, 139-155.

[4] Carreras, F. and Freixas, J. (1999). Some theoretical reasons for using regular semivalues. In Logic, Game Theory and Social Choice (Proc. Internat. Conf. LGS), Tilburg, The Netherlands, pp. 140-154.

[5] Coleman, J. S. (1971). Control of collectivities and the power of a collectivity to act. In Social Choice, ed. B. Lieberman, Gordon and Breach, New York, pp. 269-300.

[6] Dubey, P. And Shapley, L. S. (1979). Mathematical properties of the Banzhaf power index. Math. Operat. Res. 4, 99-131.

[7] Dubey, P., Neyman, P. And Weber, R. J. (1981). Value theory without efficiency. Math. Operat. Res. 6, 122-128.

[8] Felsenthal, D. S. and Machover, M. (1998). The Measurement of Voting Power. Theory and Practice, Problems and Paradoxes. Edward Elgar, Cheltenham.

[9] Freixas, J. and Pons, M. (2005). Two measures of circumstantial power: influences and bribes. Homo Oeconomicus 22, 569-588.

[10] Freixas, J. and Pons, M. (2008). Circumstantial power: optimal persuadable voters. Europ. J. Operat. Res. 186, 1114-1126.

[11] Freixas, J. And Puente, M. A. (2002). Reliability importance of the components in a system based on semivalues and probabilistic values. Ann. Operat. Res. 109, 331-342.

[12] Hammer, P. L. And Holzman, R. (1992). Approximations of pseudoboolean functions; applications to game theory. Z. Operat. Res. 36, 3-21.

[13] Isbell, J. R. (1956). A class of majority games. Quart. J. Math. Oxford Ser. 7, 183-187.

[14] Laruelle, A. and Valenciano, F. (2005). A critical reappraisal of some voting power paradoxes. Public Choice, 125, 17-41.

[15] Laruelle, A., Martínez, R. and Valenciano, F. (2006). Success versus decisiveness, conceptual discussion and case study. J. Theoret. Politics 18, 185-205.

[16] Owen, G. (1972). Multilinear extensions of games. Manag. Sci. 18, 64-79.

[17] Owen, G. (1975). Multilinear extensions and the Banzhaf value. Naval Res. Logistics Quart. 22, 741-750.

[18] Owen, G. (1995). Game Theory, 3rd edn. Academic Press, San Diego, CA.

[19] Shapley, L. S. (1962). Simple games: an outline of the descriptive theory. Behavioral Sci. 7, 59-66.

[20] Straffin, P. D. (1988). The Shapley-Shubik and Banzhaf power indices. In The Shapley Value: Essays in Honor of Lloyd S. Shapley, ed. A. E. Roth, Cambridge University Press, pp. 71-81.

[21] Taylor, A. D. and Zwicker, W. S. (1999). Simple Games: Desirability Relations, Trading, and Pseudoweightings. Princeton University Press.

[22] Weber, R. J. (1988). Probabilistic values for games. In Contributions to the Theory of Games II, eds A. W. Tucker and H. W. Kuhn, Princeton University Press, pp. 101-119. 